\title{
Prevalence of Psychotropic Polypharmacy and Associated Healthcare Resource Utilization during Initial Phase of Care among Adults with Cancer in USA
}

\author{
Hilary A. Aroke ${ }^{1}$ Ami M. Vyas ${ }^{1} \cdot$ Ashley L. Buchanan ${ }^{1} \cdot$ Stephen J. Kogut ${ }^{1}$
}

Published online: 24 April 2019

(c) The Author(s) 2019

\begin{abstract}
Background The use of psychotropic medications is not uncommon among patients with newly diagnosed cancer. However, the impact of psychotropic polypharmacy on healthcare utilization during the initial phase of cancer care is largely unknown. Methods We used a claims database to identify adults with incident breast, prostate, lung, and colorectal cancers diagnosed during 2011-12. Psychotropic polypharmacy was defined as concurrent use of two or more psychotropic medication classes for at least 90 days. A multivariable logistic regression was performed to identify significant predictors of psychotropic polypharmacy. Multivariable Poisson and negative binomial regressions were used to assess the associations between psychotropic polypharmacy and healthcare utilization.

Results Among 5604 patients included in the study, 52.6\% had breast cancer, $30.6 \%$ had prostate cancer, $11.4 \%$ had colorectal cancer, and 5.5\% had lung cancer. During the year following incident cancer diagnosis, psychotropic polypharmacy was reported in $7.4 \%$ of patients, with the highest prevalence among patients with lung cancer (14.4\%). Compared with patients without psychotropic polypharmacy during the initial phase of care, patients with newly diagnosed cancer with psychotropic polypharmacy had a 30\% higher rate of physician office visits, an 18\% higher rate of hospitalization, and a 30\% higher rate of outpatient visits. The rate of emergency room visits was similar between the two groups.

Conclusion Psychotropic polypharmacy during the initial phase of cancer care was associated with significantly increased healthcare resource utilization, and the proportion of patients receiving psychotropic polypharmacy differed by type of cancer. Impact Findings emphasize the importance of evidence-based psychotropic prescribing and close surveillance of events causing increased healthcare utilization among patients with cancer receiving psychotropic polypharmacy.
\end{abstract}

An abstract from a part of this study was presented as a poster at the 2018 Internal Conference on Pharmacoepidemiology and Therapeutic Risk Management, Prague, Czech Republic.

Electronic supplementary material The online version of this article (https://doi.org/10.1007/s40801-019-0153-5) contains supplementary material, which is available to authorized users.

Ami M. Vyas

avyas@uri.edu

1 Department of Pharmacy Practice, College of Pharmacy, University of Rhode Island, 7 Greenhouse Road, Kingston, RI 02881, USA

\section{Key Points}

Prevalence of psychotropic polypharmacy varied by type of cancer and approximately $7.4 \%$ of patients with cancer received psychotropic polypharmacy during the first year following cancer diagnosis.

Psychotropic polypharmacy was significantly associated with higher rates of physician office visits, outpatient visits, and inpatient visits.

\section{Introduction}

More than 1.5 million new cases of cancer are diagnosed each year in USA [1] and mental health and adjustment disorders are common among these patients $[2,3]$. It is 
estimated that about $50 \%$ of patients diagnosed with cancer experience clinically significant psychosocial distress and mental health disorders that may have a negative impact on their quality of life [2, 4-6]. The Institute of Medicine considers psychosocial care an essential part of good-quality cancer care and recent treatment guidelines have suggested the integration of psychosocial support and mental health services into the routine care of patients with cancer [7, 8]. Cancer by itself and its treatment may precipitate several cancer-related symptoms such as fatigue, pain, anorexia, sleep disturbances, and cognitive dysfunction [9]. Psychotropic medications are widely used to treat these cancer-related symptoms [10]. As a result, psychotropic medications have become an important tool in a multidisciplinary approach to mental health issues related to cancer care [11]. These medications are often prescribed for both psychiatric and non-psychiatric cancer symptoms [12-18] by psychiatrists, oncologists, and primary care physicians, especially around the time of diagnosis and initial phase of cancer care (i.e., the first year following cancer diagnosis) [19-21].

In routine medical practice, psychotropic polypharmacy (PP), the concomitant use of two or more classes of psychotropic medications in one patient, is not uncommon [22]. Although each drug may have an appropriate indication, this practice has received much attention in the last decade because of increased pill burden, increased healthcare cost, and safety concerns related to drug-drug interactions not only between psychotropic medications, but also between psychotropic medications and anticancer medications [23-27]. Accordingly, the annual direct cost of cancer care in USA is expected to rise from US $\$ 104$ billion in 2006 to about US $\$ 173$ billion by 2020 with a corresponding increase in healthcare resource utilization $[28,29]$. A few studies have described the use of psychotropic medications in patients with cancer [17-21, 30-32] but none have examined the impact of PP on healthcare resource utilization during the initial phase of cancer care when initial courses of cancer treatment including adjuvant therapy are usually expected to be completed. This period of active cancer treatment is characterized by significant psychosocial distress, psychotropic medication use, and frequent clinical encounters. Prior studies have shown healthcare resource utilization and cancerrelated costs to vary by several factors including cancer type, stage at diagnosis, sex, and area of residence [33, 34] with the greatest expenditure occurring among those with metastatic cancer, especially during the last year of life [34-36]. The purpose of the study was to examine the prevalence of PP, and the relationship between PP and healthcare resource utilization during the year following the initial diagnosis of breast, prostate, lung, and colorectal cancers.

\section{Methods}

\subsection{Study Design and Data Source}

This cross-sectional observational study was conducted using an administrative claims database, Optum Clinformatics ${ }^{\circledR}$ Data Mart (OptumInsight, Eden Prairie, MN, USA), covering about 22 million enrollees across USA from 2010 to 2013. Beneficiaries included in the database had both medical and prescription drug coverage. The database included demographic and enrollment information as well as medical and pharmacy claims. The inpatient file captured information on counts of hospitalization and lengths of hospital stays. The database is licensed to the University of Rhode Island, College of Pharmacy and the study protocol was approved by the Institutional Review Board of the University of Rhode Island (Kingston, RI, USA).

\subsection{Study Population}

The study was limited to breast, prostate, lung, and colorectal cancers, which represent the most common cancers among adults in USA and accounted for $46.3 \%$ (US $\$ 48$ billion) of the national economic burden of cancer care in 2006 [29, 37]. We used the following International Classification of Diseases, Ninth Revision, Clinical Modification (ICD-9-CM) codes to identify cancer cases diagnosed between 1 January, 2011 and 31 December, 2012: 174.xx, 175.xx $233.0 x$ for breast, $185 . x x, 233.4 x$ for prostate, 153.xx 154.xx, 230.3x, 230.4x for colorectal, and 162.xx, $231.2 x$ for lung cancer [38]. Each patient was required to have at least two separate medical claims with the same cancer diagnosis having service dates at least 30 days apart. The index date was defined as the date of service for the initial cancer diagnosis. To allow for adequate ascertainment of baseline characteristics and subsequent assessment of study outcomes, the analysis was restricted to patients with continuous enrollment having both medical and pharmacy benefits for 12 months before and after the index date. The baseline period was defined as the 12 months preceding the cancer diagnosis and healthcare resource utilization was assessed during the initial phase of cancer care. We also excluded patients aged younger than 18 years, female patients with any claims for prostate cancer, male patients with claims for breast cancer, and patients with more than one type of cancer or claim for metastatic cancer (ICD-9-CM: 196.xx to 198.x) at baseline or during the initial phase of cancer care. We excluded patients with any known metastatic disease because it is not possible to differentiate de novo metastatic cancer 
from a metastasis occurring following primary incident cancer diagnosis using claims data without a chart review. The flow chart for the study cohort selection process is shown in Table 1.

\subsection{Study Measures}

\subsubsection{Psychotropic Polypharmacy}

Psychotropic prescription information was obtained from outpatient pharmacy claims and categorized using the American Hospital Formulary Service Pharmacologic Therapeutic Classification System therapeutic class codes. Psychotropic medication were categorized by drug class according to their standard use as antidepressants, antipsychotics, anticonvulsants, anxiolytics-sedatives-hypnotics (including barbiturates), and central nervous system stimulants/psychostimulants. These classes of psychotropic medications were selected based on published reviews about the use of psychotropic medications among patients with cancer $[39,40]$. Psychotropic medication use was defined as the receipt of at least one prescription in the psychotropic medication classes during the study period. Psychotropic polypharmacy was defined as the concurrent use of two or more psychotropic medication classes for at least 90 days [25, 41, 42].

\subsubsection{Healthcare Resource Utilization}

Healthcare Common Procedure Coding System procedure codes, place of service, and revenue codes were used to identify specific types of healthcare resource utilization including office visits, outpatient visits, and emergency room (ER) visits during the initial phase of care. We used the inpatient data file to identify inpatient admissions and length of inpatient stay during the initial phase of care. Multiple claims with the same procedure code, place of service, and claim date were considered duplicates and only one claim was considered for assessing healthcare utilization. Each claim was treated as a distinct medical encounter, visit, or event. Office visits included claims for services performed in a physician's office while outpatient visits included encounters outside a doctor's office for which a patient is able to return home after care without an overnight stay including visits to hospital outpatient departments, oncology clinics, ambulatory care surgical centers, outpatient rehabilitation facilities, rural health clinics, and community mental health centers. Office visits were identified with procedure codes 99201-99205 and 99211-99215; outpatient visits with procedure codes 99241-9245, 99341-99350, 99381-99387, 99391-99397, 99401-99404, 99411, 99412, 99420, 99429, 99455, and 99456; and ER visits with procedure codes 99281-99288.

\subsubsection{Covariates}

Demographic characteristics obtained from the membership file included age at initial cancer diagnosis, sex, type of health plan (point of service, exclusive provider organization, health maintenance organization, and others), and US geographic census region (Northeast, South, Midwest, and West). Clinical characteristics consisted of the type of cancer (breast, prostate, lung, colorectal), Charlson comorbidity indices, psychiatric comorbidities, and chemotherapy and radiation therapy; the latter were captured with ICD9-CM codes V58.11 and V58.0, respectively. Charlson comorbidity indices were calculated for each patient based on the presence of claims for certain diagnosis during the year prior to cancer diagnosis [43]. In addition to dementia being included in the Charlson comorbidity measure, we also controlled additional baseline psychiatric conditions such as depression, anxiety, dysthymia, post-traumatic stress disorder, bipolar disorder, personality disorder, schizophrenia, and other psychotic disorders [32, 44, 45]. These were obtained from the medical file using their respective ICD9-CM codes.

\subsection{Statistical Analysis}

Descriptive statistics were computed for baseline demographic and clinical characteristics and differences between patients with and without PP during the initial phase of care were compared using Chi-square goodness-of-fit tests (for categorical variables) and $t$ tests (for continuous variables). The prevalence of PP overall and by type of cancer were also estimated and compared using $t$ tests. The mean number of prescriptions for psychotropic medications prescribed to patients and the prevalence of PP during the baseline period

Table 1 Study cohort selection

\begin{tabular}{ll}
\hline$N$ & Criteria met \\
\hline 26,064 & Claims for one type of cancer between 1/1/2011 and 31/12/2012 \\
15,292 & Continuous enrolled for 1 year before and after cancer diagnosis \\
15,279 & After excluding male with female individuals with cancers and vice versa \\
14,733 & After excluding patients with metastatic cancer during initial phase of care \\
14,176 & After excluding patients aged younger than 18 years \\
5604 & Patients with breast, prostate, lung, and colorectal cancers only \\
\hline
\end{tabular}


and initial phase of care overall and by type of cancer were estimated using $t$ tests. To identify potential predictors of PP among patients with cancer, first, a multivariable logistic regression model was conducted.

The frequency and rates of office visits, outpatient visits, ER visits, inpatient admissions, and length of inpatient stay during the initial phase of cancer care were determined. The unadjusted cumulative incidence rate for each type of healthcare resource utilization was computed as the sum of all events or the total number of patients who experienced the incident event of interest per cohort divided by the total person-time at risk for the entire cohort [46]. Second, multivariable regressions were performed to evaluate if PP vs. no PP was associated with each component of healthcare resource utilization. Adjusted incidence rate ratios (aIRRs) were estimated using either a Poisson (number of admissions) or a negative binomial (number of outpatient visits, office visits, ER visits, and hospital length of stay) regression based on a model fit for our data, after controlling for all the covariates (Appendix 1 of the Electronic Supplementary Material [ESM]). A goodness-of-fit Chi-square test was used to assess the fit of each model assuming that the deviance follows a Chi-square distribution with degrees of freedom equal to the model residual [47] (eTable 3 of the ESM).

All statistical analyses were two sided and conducted at the 0.05 significance level. Statistical analyses were performed using the Statistical Analysis Systems software (SAS ${ }^{\circledR}$ Version 9.4, SAS Institute Inc., Cary, NC, USA).

\section{Results}

\subsection{Study Population and Baseline Characteristics}

A total of 5604 patients with newly-diagnosed cancer were included in the study, of which $52.6 \%$ had breast cancer, $30.6 \%$ had prostate cancer, $11.4 \%$ had colorectal cancer, and $5.5 \%$ had lung cancer. Four hundred and sixteen patients (7.4\%) received PP during the initial phase of cancer care (Table 2). Overall, more than $77 \%$ of patients were between the ages of 45 and 64 years, $61 \%$ were female, $74.6 \%$ had a point of service health plan, and $68 \%$ received medical care in the Southern US census region. A majority of these patients had a Charlson comorbidity score of 0 (66.5\%) or did not have any diagnoses for psychiatric conditions (93.8\%) at baseline. The distributions of clinical and demographic characteristics among patients who received PP during the year following cancer diagnosis were significantly different from those without PP, except for type of health plan. At baseline, a majority of patients prescribed PP were more likely to be female (81.3\%) aged 55-64 years (56.5\%) with breast cancer $(65.1 \%)$ as compared to those without PP. Furthermore, patients who received PP during the initial phase of care were more likely to have a Charlson comorbidity index of at least 1 and mental illness before cancer diagnosis compared with patients who did not receive PP. A total of 39,001 psychotropic medication prescriptions were filled during the 2-year period around initial cancer diagnoses, of which 20,970 (53.8\%) were prescribed during the initial phase of care, 18,336 (47\%) were antidepressants, and 13,252 (34\%) were anxiolytics (eTable 1 of the ESM).

\subsection{Prevalence and Predictors of Psychotropic Polypharmacy}

The proportion of patients who received PP during the 1 year prior to cancer diagnosis increased among all types of cancers during the initial phase of cancer care (Table 3 ). On average, the proportion of patients on PP increased significantly from $6.2 \%$ before to $7.4 \%$ after cancer diagnosis. More than $90 \%$ of patients who received PP during the initial phase of care had an antidepressant as a component of their PP combination (eTable 2 of the ESM). Use of PP during the initial phase of care differed by cancer type, with patients with lung cancer (14.4\%) more likely to have PP compared with patients with breast $(9.2 \%)$, prostate $(2.9 \%)$, or colorectal $(8.0 \%)$ cancers. There was a corresponding increase in the average number of psychotropic drug prescriptions during the 12 months preceding the index date compared with the 12 months following the initial cancer diagnosis for all types of cancers, except for a relatively small but statistically non-significant decrease among patients with lung cancer (Table 4). For instance, the mean number of psychotropic drug prescriptions among patients with colorectal cancer increased from 7.95 (standard deviation $=8.97$ ) at baseline to 8.91 (standard deviation $=8.90$ ) during the initial phase of care.

Sex, age group at cancer diagnosis, presence of psychiatric conditions, and physical comorbidity at baseline were significant predictors of the use of PP during the initial phase of cancer care (Table 5). After controlling for other variables in the model, patients aged 55-64 years were more likely to receive PP compared with those aged 18-44 years (adjusted odds ratio $[\mathrm{aOR}]=1.69 ; 95 \%$ confidence interval [CI] 1.07-2.65); female patients were two times more likely to receive $\mathrm{PP}$ than male patients $(\mathrm{aOR}=2.15 ; 95 \% \mathrm{CI}$ 1.32-3.49); and patients with cancer residing in the South were 1.5 times more likely to use PP than those residing in the Northeast $(\mathrm{aOR}=1.51 ; 95 \%$ CI $1.09-2.08)$. In addition, patients with cancer with one or more psychiatric diagnostic conditions $(\mathrm{aOR}=8.39$; 95\% CI $6.32-11.19$ for one condition; $\mathrm{aOR}=11.19$; $95 \%$ CI $6.42-19.52$ for two or more conditions) were more likely to receive PP compared with those without any psychiatric condition at baseline. Finally, patients with cancer with at least one comorbidity score $(\mathrm{aOR}=1.70 ; 95 \%$ CI $1.32-2.18$ for one score; $\mathrm{aOR}=2.21$; 
Table 2 Baseline characteristics of patients diagnosed with breast, prostate, lung, and colorectal cancers in USA $(n=5604)$

\begin{tabular}{|c|c|c|c|c|}
\hline Characteristics & $\begin{array}{l}\text { Overall } \\
n=5604\end{array}$ & $\begin{array}{l}\text { No PP } \\
n=5188(92.6 \%)\end{array}$ & $\begin{array}{l}\mathrm{PP} \\
n=416(7.4 \%)\end{array}$ & $P$ value \\
\hline \multicolumn{5}{|c|}{ Age group at index (years) } \\
\hline $18-44$ & $408(7.28)$ & $382(7.36)$ & $26(6.25)$ & \multirow[t]{4}{*}{0.0202} \\
\hline $45-54$ & 1505 (26.86) & $1396(26.91)$ & $109(26.20)$ & \\
\hline $54-64$ & $2822(50.36)$ & 2587 (49.87) & $235(56.49)$ & \\
\hline $65+$ & $869(15.51)$ & $823(15.86)$ & $46(11.06)$ & \\
\hline \multicolumn{5}{|l|}{ Sex } \\
\hline Female & $3418(60.99)$ & $3080(59.37)$ & $338(81.25)$ & \multirow[t]{2}{*}{$<0.0001$} \\
\hline Male & $2186(39.01)$ & $2108(40.63)$ & $78(18.75)$ & \\
\hline \multicolumn{5}{|c|}{ Type of health plan } \\
\hline EPO & $727(12.97)$ & $679(13.09)$ & $48(11.54)$ & \multirow[t]{4}{*}{0.0748} \\
\hline HMO & $295(5.26)$ & $277(5.34)$ & $18(4.33)$ & \\
\hline Others $^{\mathrm{a}}$ & $401(7.16)$ & $359(6.92)$ & $42(10.10)$ & \\
\hline POS & $4181(74.61)$ & $3873(74.65)$ & $308(74.04)$ & \\
\hline \multicolumn{5}{|c|}{ Geographic region } \\
\hline Midwest & $1582(28.23)$ & $1466(28.26)$ & $116(27.88)$ & \multirow[t]{4}{*}{0.0500} \\
\hline Northeast & $1042(18.60)$ & $978(18.85)$ & $64(15.38)$ & \\
\hline South & $2265(40.40)$ & 2073 (39.97) & $192(46.15)$ & \\
\hline West & $714(12.74)$ & $670(12.92)$ & $44(10.58)$ & \\
\hline \multicolumn{5}{|l|}{ Type of cancer } \\
\hline Breast & $2946(52.57)$ & $2675(51.56)$ & $271(65.14)$ & \multirow[t]{4}{*}{$<0.0001$} \\
\hline Colorectal & $636(11.35)$ & $585(11.28)$ & $51(12.26)$ & \\
\hline Lung & $306(5.46)$ & $262(5.05)$ & $44(10.58)$ & \\
\hline Prostate & $1716(30.62)$ & $1666(32.11)$ & $50(12.02)$ & \\
\hline \multicolumn{5}{|c|}{ No. of psychiatric conditions } \\
\hline None & $5256(93.79)$ & $4967(65.74)$ & $289(69.47)$ & \multirow[t]{3}{*}{$<0.0001$} \\
\hline 1 & $288(5.14)$ & $189(3.64)$ & $99(23.80)$ & \\
\hline $2+$ & $60(1.07)$ & $32(0.62)$ & $28(6.73)$ & \\
\hline \multicolumn{5}{|c|}{ Charlson Comorbidity Index } \\
\hline 0 & $3729(66.54)$ & $3513(67.71)$ & $216(51.92)$ & \multirow[t]{3}{*}{$<0.0001$} \\
\hline 1 & $1252(22.34)$ & $1129(21.76)$ & $123(29.57)$ & \\
\hline $2+$ & $623(11.12)$ & $546(10.52)$ & $77(18.57)$ & \\
\hline
\end{tabular}

$E P O$ exclusive provider organization, $H M O$ health maintenance organization, $P O S$ point of service, $P P$ psychotropic polypharmacy

${ }^{a}$ Others include indemnity and preferred provider organizations
Table 3 Distribution of psychotropic polypharmacy among adults with common cancer types in USA

\begin{tabular}{llcc}
\hline Cancer types & $\begin{array}{l}\text { Total population, } n \\
(\%)\end{array}$ & Baseline, $n(\%)$ & $\begin{array}{l}\text { Initial phase } \\
\text { of care, } n(\%)\end{array}$ \\
\hline Breast & $2946(52.57)$ & $229(7.77)$ & $271(9.20)$ \\
Colorectal & $636(11.35)$ & $42(6.60)$ & $51(8.02)$ \\
Lung & $306(5.46)$ & $30(9.80)$ & $44(14.38)$ \\
Prostate & $1716(30.62)$ & $44(2.56)$ & $50(2.91)$ \\
Overall & 5604 & $345(6.16)$ & $416(7.42)$ \\
\hline
\end{tabular}

The proportion of patients with psychotropic polpharmacy at baseline and during the initial phase of care differed significantly for all cancer types (Chi square test: $p<0.0001$ )
95\% CI 1.61-3.04 for two or more scores) were more likely to have PP compared with those without any physical comorbidity at baseline.

\subsection{Rates of Healthcare Resource Utilization}

Nearly $45 \%$ of all patients had at least one outpatient and office visit, $18.5 \%$ required at least one hospital admission, and $15.7 \%$ had at least one ER visit during the initial phase of cancer care. Table 6 presents a summary of the unadjusted and adjusted rates of healthcare resource utilization among patients with cancer with and without PP during the initial phase of cancer care. After adjusting for age at cancer diagnosis, sex, type of health plan, geographic region, cancer 
Table 4 Average number of prescriptions of psychotropic medications among adults ${ }^{\mathrm{a}}$ with common cancer types in USA

\begin{tabular}{lccccc}
\hline Cancer types & At baseline & & & \multicolumn{2}{l}{ During the initial phase of care } \\
\cline { 2 - 3 } & $N(\%)$ & Mean $(95 \% \mathrm{CI})$ & & $N(\%)$ & Mean $(95 \% \mathrm{CI})$ \\
\hline Breast & $1388(62.21)$ & $8.45(7.98-8.92)$ & & $1518(63.28)$ & $9.00(8.53-9.47)$ \\
Colorectal & $231(10.35)$ & $7.95(6.79-9.11)$ & & $262(10.92)$ & $8.91(7.83-10.00)$ \\
Lung & $159(7.13)$ & $10.14(8.49-11.78)$ & & $181(7.54)$ & $10.03(8.50-11.55)$ \\
Prostate & $453(20.30)$ & $6.30(5.63-6.96)$ & & $438(18.26)$ & $7.22(6.53-7.92)$ \\
Overall & $2231(100)$ & $8.08(7.72-8.45)$ & & $2399(100)$ & $8.74(8.38-9.11)$ \\
\hline
\end{tabular}

CI confidence interval

The mean number of psychotropic medications used at baseline and during the initial phase of cancer care did not differ significantly both overall and for each type of cancer ( $t$ test: $p>0.05$ )

${ }^{a}$ Among those with at least one psychotropic medication prescription during baseline or during the initial phase of care type, number of psychiatric conditions and Charlson comorbidity index at baseline, and use of radiation and chemotherapy during the initial phase of cancer care, patients who received PP had about a 30\% higher rate of both outpatient and physician office visits (aIRR $=1.28 ; 95 \%$ CI $1.21-1.36$ and aIRR $=1.30 ; 95 \%$ CI 1.23-1.38, respectively), $18 \%$ higher rates of hospital admissions $(\mathrm{aIRR}=1.18 ; 95 \% \mathrm{CI}$ 1.07-1.31), and a 30\% increase in the length of hospital inpatient stays (aIRR $=1.30 ; 95 \%$ CI 1.11-1.52), than those without PP. The rates of ER visits were $4 \%$ higher among patients who received PP than those who did not receive PP but this difference was not statistically significant. A more detailed result of the adjusted models estimated using the Poisson and negative binomial regressions is provided in eTable 3 of the ESM.

\section{Discussion}

Several cancer- or cancer treatment-related symptoms are managed with psychotropic medications [9, 10, 19-21, 48]. In certain clinical situations, the use of more than one psychotropic medication is appropriate and supported by empirical evidence. For example, the addition of antipsychotics to either antidepressants for the treatment of major depression with psychotic features [49] or to mood stabilizers for the management of acute mania [50] is considered the standard of care. Similarly, the short-term use of benzodiazepines with antidepressants to treat major depression may be clinically justified [51]. Furthermore, patients with treatmentresistant disease may require dual therapy, and for many patients, depression, anxiety, pain, and psychosis may coexist, warranting the use of multiple psychoactive medications.

We examined the prevalence of PP, predictors of the use of PP during the initial phase of cancer care, and the association between the use of PP and healthcare resource utilization. We found that the proportion of patients who received PP increased slightly from the baseline period to the initial phase of cancer care among all patients and for all cancer types included in our study. Prevalence of psychotropic medication use and PP at baseline were relatively low $(6.2 \%)$, likely because most patients in our cohort did not have any psychiatric conditions during the year leading up to their first cancer diagnosis. A previous study found that the risk of mental health disorders is temporally related to the diagnostic work-up for suspected cancer with a corresponding increased use of psychotropic medications from about a month before a cancer diagnosis that peaks at about 3 months after [52]. This may explain the modest overall increase in the prevalence of PP use from $6.2 \%$ at baseline to $7.4 \%$ after cancer diagnosis in our study sample.

In this study, we found that in addition to the number of psychiatric conditions at baseline, sex, age at cancer diagnosis, and physical comorbidity at baseline were also significant predictors of the use of PP during the initial phase of cancer diagnosis. Type of cancer was not a significant predictor of PP use during the initial phase of cancer care. This finding is partially similar to that reported in a previous study that showed no significant association between type of cancer and the use of antidepressants [53]. Other studies have found a similarly higher prevalence of psychotropic medication use and PP among women and patients with comorbid conditions [21, 30, 54].

A higher prevalence of PP was observed among patients with lung, colorectal, and breast cancers compared with those with prostate cancer. These findings are consistent with those reported in an analysis of self-reported medication use among survivors of patients with breast, lung, and colorectal cancers [30]. A retrospective cohort study of community-based Medicare beneficiaries also found that patients with prostate cancer were least likely to receive any psychotropic medications compared with patients with breast, lung, and colorectal cancers [55]. One potential reason for a lower prevalence of PP among patients with prostate cancer is that a diagnosis of prostate cancer may be associated with less psychosocial distress requiring pharmacotherapy because a 
Table 5 Factors associated with use of psychotropic polypharmacy among adults with common cancer types during the year following cancer diagnosis in USA

\begin{tabular}{|c|c|c|}
\hline Variables & Unadjusted OR (95\% CI) & Adjusted OR (95\% CI) \\
\hline \multicolumn{3}{|c|}{ Age group at index (years) } \\
\hline $18-44$ & Ref. & Ref \\
\hline $45-54$ & $1.15(0.74-1.79)$ & $1.25(0.78-2.00)$ \\
\hline $54-64$ & $1.34(0.88-2.03)$ & $1.69(1.07-2.65)$ \\
\hline $65+$ & $0.82(0.50-1.35)$ & $1.13(0.65-1.97)$ \\
\hline \multicolumn{3}{|l|}{ Sex } \\
\hline Male & Ref. & Ref \\
\hline Female & $2.97(2.30-3.82)$ & $2.15(1.32-3.49)$ \\
\hline \multicolumn{3}{|c|}{ Type of health plan } \\
\hline HMO & Ref. & Ref. \\
\hline EPO & $1.09(0.62-1.90)$ & $1.03(0.57-1.87)$ \\
\hline Others $^{\mathrm{a}}$ & $1.80(1.01-3.20)$ & $1.82(0.98-3.39)$ \\
\hline POS & $1.22(0.75-2.00)$ & $1.27(0.76-2.14)$ \\
\hline \multicolumn{3}{|c|}{ Geographic region } \\
\hline Northeast & Ref. & Ref. \\
\hline Midwest & $1.21(0.88-1.66)$ & $1.26(0.89-1.77)$ \\
\hline South & $1.42(1.06-1.90)$ & $1.51(1.09-2.08)$ \\
\hline West & $1.00(0.678-1.49)$ & $1.13(0.74-1.73)$ \\
\hline \multicolumn{3}{|c|}{ Type of cancer } \\
\hline Breast & Ref. & Ref. \\
\hline Colorectal & $0.86(0.630-1.18)$ & $1.29(0.89-1.88)$ \\
\hline Lung & $1.66(1.176-2.34)$ & $1.46(0.96-2.22)$ \\
\hline Prostate & $0.30(0.218-0.40)$ & $0.62(0.35-1.12)$ \\
\hline \multicolumn{3}{|c|}{ Chemotherapy } \\
\hline No & Ref. & Ref. \\
\hline Yes & $1.46(1.07-1.98)$ & $1.15(0.81-1.64)$ \\
\hline \multicolumn{3}{|l|}{ Radiotherapy } \\
\hline No & Ref. & Ref. \\
\hline Yes & $1.00(0.673-1.47)$ & $0.86(0.55-1.33)$ \\
\hline \multicolumn{3}{|c|}{ No. of psychiatric conditions } \\
\hline None & Ref. & Ref. \\
\hline 1 & $9.00(6.87-11.80)$ & $8.39(6.32-11.13)$ \\
\hline $2+$ & $15.04(8.93-25.32)$ & $11.19(6.42-19.52)$ \\
\hline \multicolumn{3}{|c|}{ Charlson Comorbidity Index } \\
\hline 0 & Ref. & Ref. \\
\hline 1 & $1.77(1.41-2.23)$ & $1.70(1.32-2.18)$ \\
\hline $2+$ & $2.29(1.74-3.02)$ & $2.21(1.61-3.04)$ \\
\hline
\end{tabular}

Bold values indicate $\mathrm{p}<0.05$

$C I$ confidence interval, $E P O$ exclusive provider organization, $H M O$ health maintenance organization, $O R$ odds ratio, $P O S$ point of service

${ }^{a}$ Others include indemnity and preferred provider organizations significant portion of patients with prostate cancer die with the disease rather than from the disease.

We found that the use of PP during the first year after a cancer diagnosis was associated with increased rates of outpatient visits, office visits, inpatient admissions, and length of hospital stay after adjusting for certain potential confounding factors. Patients with newly diagnosed cancer often require chemotherapy and concurrent use of multiple psychotropic medications places them at a significantly higher risk of adverse drug-drug interactions that may require additional healthcare services for their effective management [24, 27, 56]. Patients who received PP in our study were more likely to have many chronic physical and psychiatric conditions. This may explain why they experienced more frequent clinical encounters and received more psychotropic medications. To the best of 
Table 6 Unadjusted and adjusted rates of healthcare resource utilization during the initial phase of cancer care comparing patients with psychotropic polypharmacy (PP) and without PP among adults with common cancer types in USA

\begin{tabular}{lcl}
\hline $\begin{array}{l}\text { Healthcare resource } \\
\text { utilized }\end{array}$ & Unadjusted IRR & Adjusted IRR $(95 \%$ CI) \\
\hline $\begin{array}{l}\text { Number of outpatient } \\
\text { visits }\end{array}$ & $1.25(1.21-1.28)$ & $\mathbf{1 . 2 8}(\mathbf{1 . 2 1 - 1 . 3 6})$ \\
Number of office visits & $1.26(1.23-1.29)$ & $\mathbf{1 . 3 0}(\mathbf{1 . 2 3}-\mathbf{1 . 3 8})$ \\
Number of ER visits & $1.19(1.09-1.29)$ & $1.04(0.88-1.23)$ \\
Number of admissions & $1.27(1.16-1.40)$ & $\mathbf{1 . 1 8}(\mathbf{1 . 0 7 - 1 . 3 1})$ \\
Length of stay (days) & $1.34(1.25-1.43)$ & $\mathbf{1 . 3 0}(\mathbf{1 . 1 1 - 1 . 5 2})$ \\
\hline
\end{tabular}

Bold values indicate $\mathrm{p}<0.05$

$C I$ confidence interval, ER emergency room, IRR incidence rate ratio

${ }^{a}$ Adjusted for age at index, sex, type of health plan, geographic region, cancer type, psychiatric conditions, and Charlson Comorbidity Index at baseline, and use of radiation and chemotherapy during the initial phase of cancer care

the authors' knowledge, this is the first study to examine the prevalence and predictors of PP during the first year of cancer care among patients with newly diagnosed breast, prostate, lung, and colorectal cancers in USA using a large insured adult population.

Our study design has some limitations. First, multiclass PP relies on a grouping of psychotropic medications into classes that is based on certain clinical syndromes or mechanisms of action that may not fully capture their pharmacological properties. In recent years, some psychotropic medications have received approvals for multiple indications. Because we did not assess the appropriateness of PP or adverse effects, we do not know if increased healthcare utilization is directly attributable to the consequences of PP or mental healthcare. In addition, some information that may impact on the use of psychotropic medications on healthcare resource utilization was not captured in our data source. Specifically, data on variables such as race/ethnicity, socioeconomic status, clinical information about the cancer (e.g., severity, tumor stage, tumor size, and tumor grade), performance status, and patient preferences and beliefs about alternative treatments was not available in the extant database. Second, a cross-sectional design limits our ability to make causal inferences owing, in part, to lack of temporality. We also conditioned our analysis on survival during the initial phase of care and occurrence of metastatic disease measured after baseline, which could induce selection bias. As a result, the true rates of PP among patients with cancer are likely higher than our estimates because psychotropic medication use often starts during evaluations for suspected cancer and peaks after cancer diagnosis, and may persist during terminal care [21]. Patients who had more advanced cancers and died during the initial phase of care were excluded, which may have resulted in healthier patients included in our analysis, as compared to all patients with incident cancer in the database. The list of procedure codes for office visits may not be exhaustive. Last, the study focused only on a commercially insured population, hence the findings may not be generalizable to other populations.

\section{Conclusion}

Patients with PP during the initial phase of cancer care utilized more healthcare resources than those without PP. There was a small but statistically significant increase in the use of psychotropic medications and PP after initial cancer diagnosis, and the proportion of patients receiving PP differed by the type of cancer. Given current treatment guideline recommendations, this increase may be the result of a greater inclusion of a psychiatrist specialized in psycho-oncology in multidisciplinary cancer care teams, and the availability of newer psychotropic medications. Psychotropic medication use may be an indicator of better assessments of psychosocial distress associated with a new cancer diagnosis. These findings emphasize the importance of evidence-based psychotropic prescribing and close surveillance for potential adverse psychotropic drug-drug interactions in patients with cancer, and suggest that patients with cancer with mental health illnesses, even when treated, require a higher degree of healthcare services. Further research is needed to determine the proportion of increased healthcare resource utilization in this population attributable to the underlying mental health co-morbidity, or psychotropic medication side effects, interactions, or other potential adverse outcomes of therapy.

Author Contributions Conception and design: AV. Data collection: AV, HA, SK. Data analysis: AV, HA, AB. Data interpretation: AV, HA, AB, SK. Manuscript writing: HA, AV. Manuscript revision and approval of final article: $\mathrm{HA}, \mathrm{AV}, \mathrm{AB}, \mathrm{SK}$.

\section{Compliance with Ethical Standards}

Funding This study was funded by the American Association of Colleges of Pharmacy (AACP) New Investigator Award mechanism received by Ami Vyas. Hilary Aroke was partially supported by the AACP funding for conducting data analyses. Stephen Kogut is partially supported by Institutional Development Award \#U54GM115677 from the National Institute of General Medical Sciences of the National Institutes of Health, which funds Advance Clinical and Translational Research (Advance-CTR). The content of this study is solely the responsibility of the authors and does not represent the official views of the AACP or the National Institutes of Health.

Conflict of interest Hilary Aroke, Ami Vyas, Ashley Buchanan, and Stephen Kogut have no conflict of interests that are directly relevant to the content of this study.

Open Access This article is distributed under the terms of the Creative Commons Attribution-NonCommercial 4.0 International License 
(http://creativecommons.org/licenses/by-nc/4.0/), which permits any noncommercial use, distribution, and reproduction in any medium, provided you give appropriate credit to the original author(s) and the source, provide a link to the Creative Commons license, and indicate if changes were made.

\section{References}

1. Siegel RL, Miller KD, Jemal A. Cancer statistics, 2018. CA Cancer J Clin. 2018;68(1):7-30.

2. Mitchell AJ, Chan M, Bhatti H, Halton M, Grassi L, Johansen $\mathrm{C}$, et al. Prevalence of depression, anxiety, and adjustment disorder in oncological, haematological, and palliative-care settings: a meta-analysis of 94 interview-based studies. Lancet Oncol. 2011;12(2):160-74.

3. Linden W, Vodermaier A, Mackenzie R, Greig D. Anxiety and depression after cancer diagnosis: prevalence rates by cancer type, gender, and age. J Affect Disord. 2012;141(2-3):343-51.

4. Derogatis LR, Morrow GR, Fetting J, Penman D, Piasetsky S, Schmale AM, et al. The prevalence of psychiatric disorders among cancer patients. JAMA. 1983;249(6):751-7.

5. Singer S, Das-Munshi J, Brahler E. Prevalence of mental health conditions in cancer patients in acute care: a meta-analysis. Ann Oncol. 2010;21(5):925-30.

6. Mehnert A, Brahler E, Faller H, Harter M, Keller M, Schulz $\mathrm{H}$, et al. Four-week prevalence of mental disorders in patients with cancer across major tumor entities. J Clin Oncol. 2014;32(31):3540-6.

7. Page AE, Adler NE. Cancer care for the whole patient: meeting psychosocial health needs. Washington DC, USA: National Academies Press; 2008.

8. McFarland DC, Holland JC. The management of psychological issues in oncology. Clin Adv Hematol Oncol. 2016;14(12):999-1009.

9. Lee BN, Dantzer R, Langley KE, Bennett GJ, Dougherty PM, Dunn AJ, et al. A cytokine-based neuroimmunologic mechanism of cancer-related symptoms. NeuroImmunoModulation. 2004;11(5):279-92.

10. Grassi L, Riba M. Clinical psycho-oncology: an international perspective. West Sussex, UK: Wiley; 2012. p. 109-26.

11. Goldberg RJ, Cullen LO. Use of psychotropics in cancer patients. Psychosomatics. 1986;27(10):687-92, 97-700.

12. Lee R, Spencer PS. Antidepressants and pain: a review of the pharmacological data supporting the use of certain tricyclics in chronic pain. J Int Med Res. 1977;5(1 Suppl.):146-56.

13. Carasso RL, Yehuda S, Streifler M. Clomipramine and amitriptyline in the treatment of severe pain. Int J Neurosci. 1979;9(3):191-4.

14. Lindsay PG, Wyckoff M. The depression-pain syndrome and its response to antidepressants. Psychosomatics. 1981;22(7):571-3, 6-7.

15. Massie MJ, Holland JC. Diagnosis and treatment of depression in the cancer patient. J Clin Psychiatry. 1984;45(3 Pt 2):25-9.

16. Qu D, Zhang Z, Yu X, Zhao J, Qiu F, Huang J. Psychotropic drugs for the management of cancer-related fatigue: a systematic review and meta-analysis. Eur J Cancer Care (Engl). 2016;25(6):970-9.

17. Derogatis LR, Feldstein M, Morrow G, Schmale A, Schmitt M, Gates C, et al. A survey of psychotropic drug prescriptions in an oncology population. Cancer. 1979;44(5):1919-29.

18. Stiefel FC, Kornblith AB, Holland JC. Changes in the prescription patterns of psychotropic drugs for cancer patients during a 10-year period. Cancer. 1990;65(4):1048-53.
19. Desplenter F, Bond C, Watson M, Burton C, Murchie P, Lee AJ, et al. Incidence and drug treatment of emotional distress after cancer diagnosis: a matched primary care case-control study. $\mathrm{Br}$ J Cancer. 2012;107(9):1644-51.

20. Farriols C, Ferrandez O, Planas J, Ortiz P, Mojal S, Ruiz AI. Changes in the prescription of psychotropic drugs in the palliative care of advanced cancer patients over a seven-year period. J Pain Symptom Manage. 2012;43(5):945-52.

21. Ng CG, Boks MP, Smeets HM, Zainal NZ, de Wit NJ. Prescription patterns for psychotropic drugs in cancer patients; a large population study in the Netherlands. Psychooncology. 2013;22(4):762-7.

22. Karow A, Lambert M. Polypharmacy in treatment with psychotropic drugs: the underestimated phenomenon. Curr Opin Psychiatry. 2003;16(6):713-8.

23. Fick D. Potentially Inappropriate medication use in a Medicare managed care population: association with higher costs and utilization. J Manage Care Pharm. 2001;7(5):407-13.

24. Sandson NB, Armstrong SC, Cozza KL. An overview of psychotropic drug-drug interactions. Psychosomatics. 2005;46(5):464-94.

25. Correll CU, Gallego JA. Antipsychotic polypharmacy: a comprehensive evaluation of relevant correlates of a long-standing clinical practice. Psychiatr Clin North Am. 2012;35(3):661-81.

26. Stahl SM. Antipsychotic polypharmacy: evidence based or eminence based? Acta Psychiatr Scand. 2002;106(5):321-2.

27. Yap KY, Tay WL, Chui WK, Chan A. Clinically relevant drug interactions between anticancer drugs and psychotropic agents. Eur J Cancer Care (Engl). 2011;20(1):6-32.

28. Mariotto AB, Yabroff KR, Shao Y, Feuer EJ, Brown ML. Projections of the cost of cancer care in the United States: 2010-2020. J Natl Cancer Inst. 2011;103(2):117-28.

29. National Cancer Institute. Cancer trends progress report: 2017 update. National Cancer Institute, NIH, Bethesda, MD, April 2017. http://www.progressreport.cancer.gov. Accessed 25 Feb 2019.

30. Punekar RS, Short PF, Moran JR. Use of psychotropic medications by US cancer survivors. Psychooncology. 2012;21(11):1237-43.

31. Goldberg RJ, Mor V. A Survey of psychotropic use in terminal cancer patients. Psychosomatics. 1985;26(9):745-8, 751.

32. Braun IM, Rao SR, Meyer FL, Fedele G. Patterns of psychiatric medication use among nationally representative long-term cancer survivors and controls. Cancer. 2015;121(1):132-8.

33. Palmer NR, Geiger AM, Lu L, Case LD, Weaver KE. Impact of rural residence on forgoing healthcare after cancer because of cost. Cancer Epidemiol Biomark Prev. 2013;22(10):1668-76.

34. Yabroff KR, Warren JL, Brown ML. Costs of cancer care in the USA: a descriptive review. Nat Clin Pract Oncol. 2007;4(11):643-56.

35. Lubitz JD, Riley GF. Trends in Medicare payments in the last year of life. N Engl J Med. 1993;328(15):1092-6.

36. Fireman BH, Quesenberry CP, Somkin CP, Jacobson AS, Baer $\mathrm{D}$, West $\mathrm{D}$, et al. Cost of care for cancer in a health maintenance organization. Health Care Financ Rev. 1997;18(4):51-76.

37. American Cancer Society. Cancer facts and figures 2018. Atlanta, GA. https://www.cancer.org/content/dam/cancer-org/research/ cancer-facts-and-statistics/annual-cancer-facts-and-figures/2018/ cancer-facts-and-figures-2018.pdf. Accessed 12 Nov 2018.

38. Chastek B, Harley C, Kallich J, Newcomer L, Paoli CJ, Teitelbaum AH. Health care costs for patients with cancer at the end of life. J Oncol Pract. 2012;8(6):75-80.

39. Thekdi SM, Trinidad A, Roth A. Psychopharmacology in cancer. Curr Psychiatry Rep. 2015;17(1):529.

40. Caruso R, Grassi L, Nanni MG, Riba M. Psychopharmacology in psycho-oncology. Curr Psychiatry Rep. 2013;15(9):393.

41. Edelsohn GA, Schuster JM, Castelnovo K, Terhorst L, Parthasarathy M. Psychotropic prescribing for persons with 
intellectual disabilities and other psychiatric disorders. Psychiatr Serv. 2014;65(2):201-7.

42. Mojtabai R, Olfson M. National trends in psychotropic medication polypharmacy in office-based psychiatry. Arch Gen Psychiatry. 2010;67(1):26-36.

43. Klabunde CN, Potosky AL, Legler JM, Warren JL. Development of a comorbidity index using physician claims data. J Clin Epidemiol. 2000;53(12):1258-67.

44. Iglay K, Santorelli ML, Hirshfield KM, Williams JM, Rhoads GG, Lin Y, et al. Diagnosis and treatment delays among elderly breast cancer patients with pre-existing mental illness. Breast Cancer Res Treat. 2017;166(1):267-75.

45. Kadan-Lottick NS, Vanderwerker LC, Block SD, Zhang B, Prigerson HG. Psychiatric disorders and mental health service use in patients with advanced cancer: a report from the coping with cancer study. Cancer. 2005;104(12):2872-81.

46. Parthan A, Santos E, Becker L, Small A, Lalla D, Brammer $\mathrm{M}$, et al. Health care utilization and costs by site of service for nonmetastatic breast cancer patients treated with trastuzumab. J Manag Care Spec Pharm. 2014;20(5):485-93.

47. Hosmer Jr DW, Lemeshow S, Sturdivant RX. Applied logistic regression. Hoboken, NJ, USA: Wiley; 2013.

48. Jones SM, Rosenberg D, Ludman E, Arterburn D. Medical comorbidity and psychotropic medication fills in older adults with breast or prostate cancer. Support Care Cancer. 2015;23(10):3005-9.

49. Wijkstra J, Lijmer J, Burger H, Cipriani A, Geddes J, Nolen WA. Pharmacological treatment for psychotic depression. Cochrane Database Syst Rev. 2015;30(7):CD004044.
50. Hirschfeld R, Bowden CL, Gitlin MJ, Keck PE, Suppes T, Thase ME, et al. Practice guideline for the treatment of patients with bipolar disorder. Arlington, VA: American Psychiatric Association; 2002.

51. Gelenberg AJ, Freeman MP, Markowitz JC, Rosenbaum JF, Thase ME, Trivedi MH, et al. Practice guideline for the treatment of patients with major depressive disorder. Third Edition. Am J Psychiatry. 2010.

52. Lu D, Andersson TM, Fall K, Hultman CM, Czene K, Valdimarsdottir U, et al. Clinical diagnosis of mental disorders immediately before and after cancer diagnosis: a nationwide matched cohort study in Sweden. JAMA Oncol. 2016;2(9):1188-96.

53. Janberidze E, Hjermstad MJ, Brunelli C, et al. The use of antidepressants in patients with advanced cancer-reults from an international multicentre study. Psychooncology. 2014;23:1096-102.

54. Lunsky Y, Modi M. Predictors of psychotropic polypharmacy among outpatients with Psychiatric disorders and intellectual disability. Psychiatr Serv. 2018;69(2):242-6.

55. Zuckerman IH, Davidoff AJ, Erten MZ, Stuart B, Shaffer T, Dougherty JS, et al. Use of and spending on supportive care medications among Medicare beneficiaries with cancer. Support Care Cancer. 2014;22(8):2185-95.

56. Chan A, Ng TR, Yap KY. Clinically-relevant anticancer-antidepressant drug interactions. Expert Opin Drug Metab Toxicol. 2012;8(2):173-99. 